\title{
Comparing Political Polarization in Political and Cultural Preferences
}

\author{
Sean Fischer*1 \\ ${ }^{1}$ Annenberg School for Communication, University of \\ Pennsylvania.
}

November 1, 2020

\begin{abstract}
The popular press has given substantial attention to the notion that Democrats and Republicans hold diverging cultural and lifestyle preferences that manifest in the TV shows they watch, the music they listen to, and the clothes they buy. The academic research in this area is split, though, with some suggesting that such divisions exist and others arguing that they ultimately fail to materialize in real-world behavior. In this study, I use network methods to evaluate whether such partisan cultural polarization exists at the individuallevel. I do so by constructing networks of shared cultural preferences and networks of shared political beliefs based on closed-ended survey responses. For each network, I calculate the assortativity (correlation) between linked respondents' partisan identity, ideology, age, gender, race, and education level. I show that the assortativity for the political identity measures is low across the cultural-preference networks compared to the political-belief networks. These results suggest that cultural preferences are not associated with partisan or ideological identities.
\end{abstract}

${ }^{*}$ Corresponding author: Sean Fischer (sean.fischer@asc.upenn.edu) 
The popular press has devoted substantial attention during and since the 2016 presidential election circulating claims about substantial divisions in the cultural and lifestyle preferences of Democrats and Republicans. These arguments build on the analyses showing aggregatelevel associations between partisanship and indicators of culture preferences, such as television viewing habits (Katz, 2016), concert attendance ("Why Obama-Trump swing voters like heavy metal", 2019), and shopping habits (Kapner \& Chinni, 2019). The notion that the parties are culturally divided has since become pervasive, with cultural preferences now operating as activators of partisan stereotypes and biases (Deichert, 2018; Hiaeshutter-Rice et al., 2019).

However, academics have not found consistent evidence to support these claims. Early work, like Bishop's seminal analysis of partisan residential sorting (Bishop, 2009), argued that partisans preferred to build and live in communities filled with like-minded others. Building on this concept, DellaPosta, Shi, and Macy conceptualized the parties as Weberian "status groups" and showed that homophily and social influence could produce within-party alignment in regards to cultural preferences (DellaPosta et al., 2015). Contrarily, Mummolo and Nall show that reported preferences for specific community attributes (e.g., more conservative) often do not translate into actual behavior changes when moving (Mummolo \& Nall, 2017). Martin and Webster support this finding by showing that partisan biases in residential choice are too small to sustain observed geographic polarization (Martin \& Webster, 2020).

It is possible, though, that individual-level psychological and sociological features correlated with partisanship may be associated with cultural and lifestyle preferences. For example, Mutz and Rao demonstrate that liberals are more likely to prefer lattes than conservatives because they are more open to globalization than conservatives (Mutz \& Rao, 2018). Similarly, Republicans' greater religiosity levels lead them to give more to charity than Democrats (Margolis \& Sances, 2017). As such, it is entirely possible that, while partisanship or ideology is not the primary pathway through which such divides emerge, the parties are effectively divided in their cultural preferences.

Clarifying whether such a divide in cultural preferences exists is crucial because of the pervasiveness of partisan cultural stereotypes. These stereotypes that link partisan groups to specific cultural habits and products are used to infer another's partisanship when obvious political signals are not available (Deichert, 2018; Hiaeshutter-Rice et al., 2019). These inferences enable people to activate their own partisan biases (Deichert, 2018), fueling affective polarization in situations that would otherwise be devoid of politics. Losing these apolitical moments is concerning because they provide opportunities for positive cross-party contact and relationships to form that could help partisans recognize similarities in the other side, leading to reductions in political hostility (Levendusky, 2018).

In this study, I take a new approach to assessing the magnitude of the partisan divide in cultural preferences. Drawing on closed-ended survey questions, I construct two sets of networks. The first set represents shared cultural preferences between the survey respondents. 
The second set represents shared political concerns between the same set of survey respondents. I then analyze each network's structure and compare the association between that structure and respondents' political identity, ideology, age, gender, race, and education level. I find that the association between network structure and respondents' political identity and ideology is substantively higher in the networks of political concerns than in the networks of shared cultural preferences. In the latter set, the association between individual-level political features and network structure is effectively zero. This result suggests that people do not encounter substantial partisan polarization in their cultural preferences.

\section{Data}

This study's data came from multiple waves of a larger study administered on Amazon Mechanical Turk (mTurk) in the Spring and Winter of 2019. Across all waves of the study, over 1,300 workers completed a survey asking about their demographics, cultural preferences, and political opinions. Descriptive statistics about the sample are provided in Appendix B.

The questions about cultural preferences were based on those used by Bourdieu in his study of cultural prestige (Bourdieu, 1984). These questions asked respondents to indicate their three favorite musical artists, television programs, literary genres, and film genres from closed lists, as well as their single favorite style of clothing from a closed list.

The questions about political beliefs and concerns included a similar question asking respondents to indicate their three most pressing political concerns from a closed list. The survey also asked respondents to indicate their specific preference for raising or lowering taxes, involvement in foreign conflicts, and a pathway to citizenship for undocumented immigrants.

All respondents also completed a set of demographic items that asked them to report the year they were born, their highest level of completed education, and how they identify in regards to gender, race and ethnicity, political party, and ideology. These last two items, political identity and ideological identity, were reported on seven-point scales. I collapsed the measure of highest educational achievement into a binary indicator for holding a college degree or not.

For the cultural-preference questions, the political issue concerns questions, and two of the political policy questions, the order of the responses was randomized to avoid order effects.

All questions and response options are included in Appendix A.

\section{Network Methodology}

Networks have been employed to study polarization in political beliefs and consumer habits. These networks break from a traditional "social" framework by studying the two-mode networks of people and ob- 


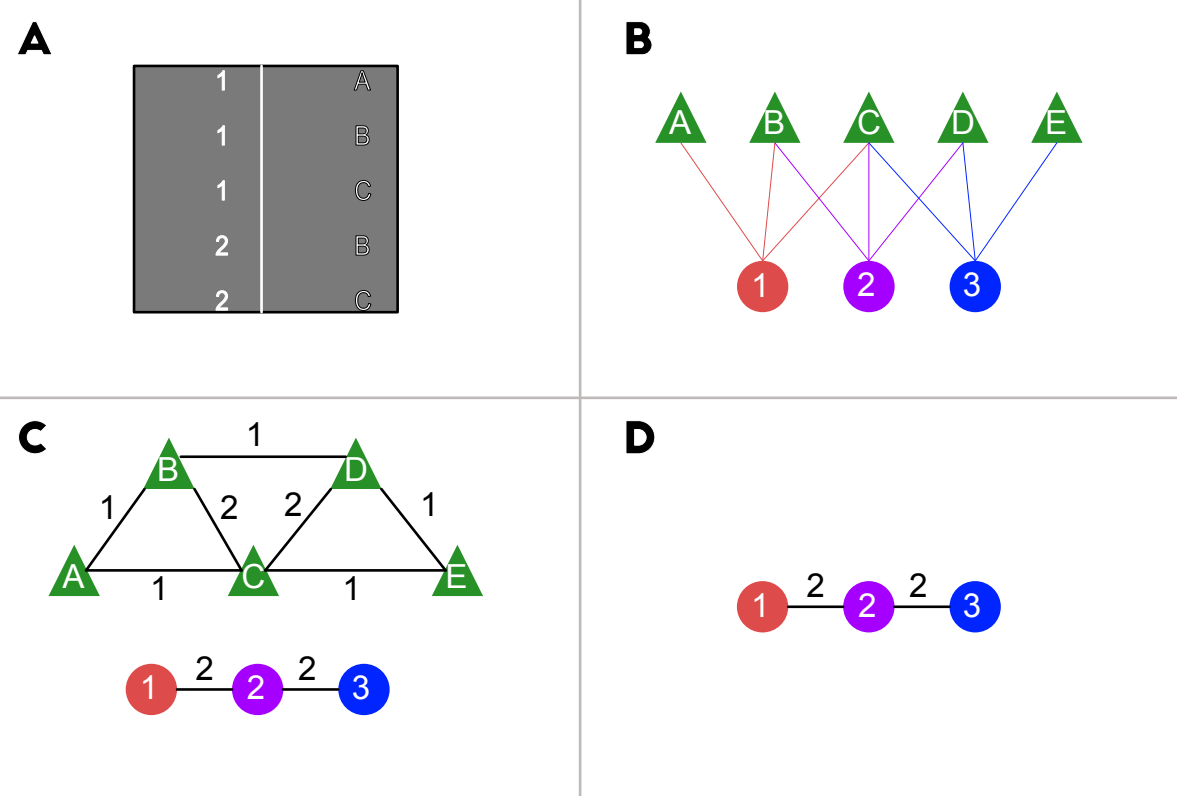

Figure 1: A. Data are generated from survey responses. B. The survey data are used to create a two-mode network linking respondents to their preferences or beliefs. C. The two-mode network is used to create two different one-mode networks. The top network maps the overlap in those who hold any two preferences. The bottom maps the shared preferences between any two respondents. D. From the two one-mode networks, I preserve the network of shared preferences between respondents.

jects. These objects may be political beliefs (DellaPosta, 2020) or cultural goods (Hoffman, 2019). In either case, the two-mode networks are converted into single-mode networks for analysis.

Traditionally, these projections have been into networks that map the ties between objects based on them being shared by multiple people. While this does allow for aggregation of features such as the average age or ideology of the people holding a belief or consuming a product, the resulting measures come with their own additional uncertainty.

To avoid this unnecessary uncertainty, I instead take my two-mode networks of people and objects (political concerns and cultural preferences) and convert them into one-mode networks of people. In these new networks, the nodes (people) are connected when they share at least one concern or preference with someone else. The resulting edges then take on a weight equal to the count of shared concerns or preferences, while the nodes are assigned their individual-level demographic features. The network generating process is visualized in Figure 1.

In these networks, the network structure, the set of connections between nodes, reflects shared political concerns and cultural preferences. As such, we may ask whether it depends on the demographic features of the nodes. More simply, this network approach allows me to 


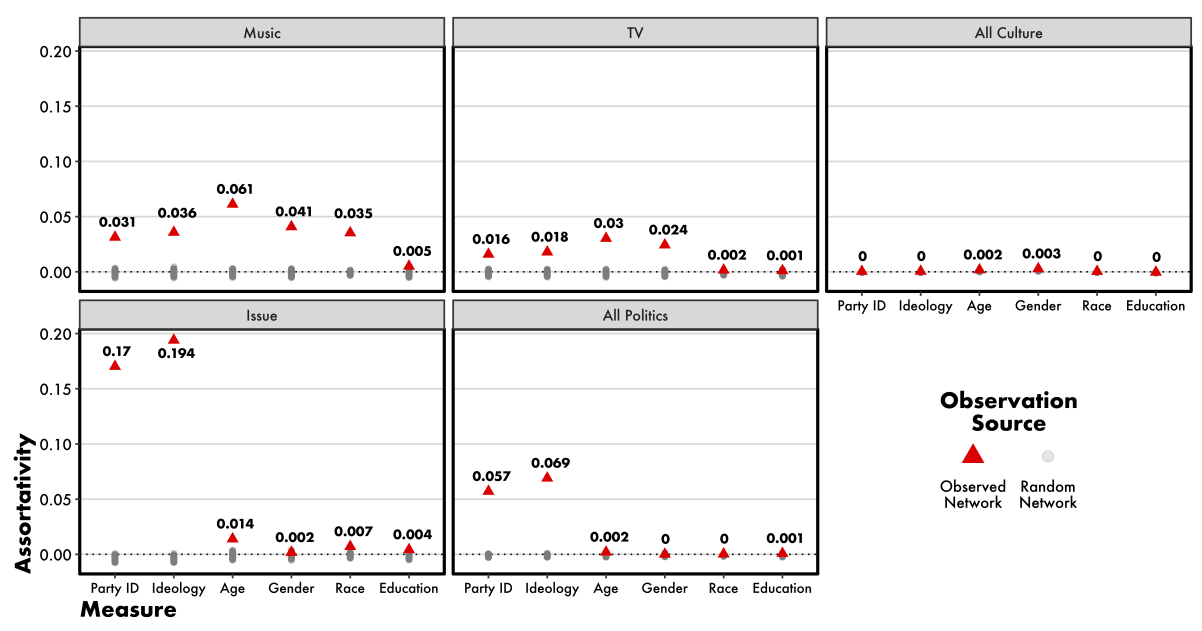

Figure 2: Assortativity by network and measure. Red triangles mark the observed assortativity for each measure in the original networks, while gray circles indicate the assortativity for each measure from a randomly rewired network with the same degree distribution.

ask whether nodes are more likely to share political concerns or cultural preferences with those who are similar to them on a range of demographic features, including political identity, ideology, age, gender, race, and education level. These associations are measured by the network assortativity. Assortativity is a correlation-like statistic ranging between -1 and 1 . Higher values indicate that nodes that are more similar on given measure are more likely to share an edge. Lower values indicate that nodes are more likely to be connected to others that are different from them on the given measure. A value close to zero indicates no relationship between the network structure and a given measure.

\section{Results}

For the set of networks mapping shared political beliefs and concerns, I constructed two specific networks. I built the first by only using data from the question asking respondents to identify their three most pressing political concerns from a closed list. Pulling from this limited set of data ensured that all ties were based on a single consistent question, which ensured that potentially differing interpretations between concerns and policy beliefs were avoided. In the second network, I included data from the question about pressing issues and three policy opinion questions about taxes, foreign conflict, and a pathway to citizenship for undocumented immigrants.

For the set of networks mapping shared cultural preferences, I built three networks. The first was based only on the data from the question about favorite musical artists. The second was based only on the data from the question about favorite television programs. The third utilized 
the data generated from all of the cultural preference questions. Again, these distinctions were made to isolate data generated from questions that could have been interpreted differently from the others.

For each network, I calculated the network assortativity based on the partisan identity, ideology, age, gender, race, and education level of each respondent in the networks. Figure 2 presents the statistics for each network based on each category, as well as a distribution of statistics for each network-measure pair that are drawn from random networks with the same degree distribution as the observed network. These observations from the random networks provide essential information about whether the observed values are extreme and go beyond what we would expect from a generating process in which the measures and the preferences were not linked.

We can see in the figure that for the three cultural-preference networks, the assortativity statistic for all five measures is low, although in some cases the statistics from the observed networks are greater than we would expect from the null model. For the music network, assortivity statistics for party identification $(r=0.031)$ and ideology $(r=0.036)$ are comparable to the statistics for age $(r=0.061)$, gender $(r=0.041)$ and race $(r=0.035)$, while the statistic for education is much lower than the other four $(r=0.005)$. In the TV network, the assortativity statistics for party identification $(r=0.016)$ and ideology $(r=0.018)$ are slightly lower than that for age $(r=0.03)$ and gender $(r=0.024)$, but are higher than those for both race $(r=0.002)$ and education $(r=0.001)$. In the network using all of the cultural-preference data, the assortativity statistics for all measures but gender drops close to zero $(r<0.01)$.

By contrast, we can see that in the political-belief networks, the assortativity statistics for party identification and ideology are both much higher than any of those for gender, race, or education. In the network built using only the respondents' three most pressing political concerns, the assortativity statistics for partisan identity $(r=0.17)$ and ideology $(r=0.19)$ reach the two highest marks across all of the observed networks. Notably, these marks, while they indicate only a weak association between the measures and the network structure, are substantively greater than any of the other relationships observed across all of the networks. Additionally, in the issue-concern network, the other four measures produce statistics close to zero, three of which fall in the null model's distribution.

When adding ties based on respondents' political opinions to the issue network, as I do in the full political-beliefs network, the assortativity statistics for partisan identity $(r=0.057)$ and ideology $(r=0.069)$ both drop substantially. However, we see that in this case the other four features all remain effectively zero and within the bands of the observations drawn from random networks. While very weak, the only features that have any association with shared political beliefs are respondents' partisan identity and ideology. 


\section{Discussion}

In this study, I constructed two sets of networks from survey responses. The first set of networks mapped shared cultural preferences for musical artists, television programs, and a combination of these two, literary genres, film genres, and clothing styles. The second set of networks mapped shared political considerations for specific issue concerns and then a combination of issue concerns and issue positions. For each network, I calculated the assortativity statistic, a measure of the correlation in linked nodes' features, for respondents' party identification, ideology, age, gender, race, and education level. Higher values for these statistics indicate greater levels of homophily, the tendency for like individuals to be connected in the network. By comparing how these statistics vary across networks, I can assess whether some preferences are more associated with political identities than others.

I find that the political networks, particularly the network based on shared issue concerns, show higher levels of political homophily and much lower levels of age-, gender-, race-, or education-based homophily than the cultural-preference networks. In these cultural-preference networks, all of the features besides education-level show very weak associations with shared preferences.

These patterns undermine claims made in the popular press and scholarly research that partisans are deeply divided in their cultural and lifestyle preferences. I show that partisans are not any more likely to share their cultural preferences with fellow in-party respondents than out-party respondents. Furthermore, my results indicate that the very weak associations observed between cultural preferences and political identities are not substantively different from those observed in regards to age, gender, and race. The lack of differentiation suggests that this very weak association is not likely to stand out in everyday experiences.

By contrast, the observed associations between political identities and shared political beliefs are likely to stand out, especially regarding issue concerns. While the observed relationships are still weak, they are substantially stronger than those observed for any other demographic feature. This difference in the magnitudes makes these relationships more likely patterns to be casually observed in everyday life.

However, this study comes with an important set of limitations. First, the networks I built and studied were constructed based on closedended survey questions. It is possible that these closed-ended questions did not properly represent the set of musical artists, television programs, political issue concerns, or policy preferences that matter to most Americans. For the cultural-preference questions, list items were chosen based on current popular artists and television programs. Other cultural-preference topics were broader, with lists composed of genre descriptors. Without examples, these descriptors may not have been well understood by respondents. While based on existing question designs used in large surveys, the political-belief questions could also have been hard to understand and interpret for respondents, leading to confusion when answering. Additionally, the issue-concern question did not provide respondents with the ability to indicate their preferred policy, if 
any, in response to the issue; Democrats and Republicans could easily have said the same issues were concerns because of prominent political debates at the time, but held drastically different opinions about how best to address the issues. While it is unclear how addressing other concerns about the questions used would affect the observed results, in this case it is likely that improving the issue concern question to include information about how to respond to those issues or which party would be better suited to addressing the issue would produce even higher levels of partisan homophily in the issue network, making the observed relationships likely lower-bounds for this empirical network.

Furthermore, generating the data used to build these networks through direct survey questions leaves open the possibility that respondents would bias their responses either through satisficing or motivated responding. In the case of satisficing, respondents may have selected the first items in the randomly ordered lists presented to them or only selected a few very popular artists because they were quickly identifiable without giving proper consideration to their preferences. It is unclear in which direction this type of behavior would bias the resulting network statistics. In the case of motivated responding, respondents may have felt the need to align their answers to these questions with their stated identities earlier in the survey. For example, Republican respondents may have felt the need not to indicate a preference for musical artists that supported Hillary Clinton in the 2016 election, like Beyoncé, even though they actually enjoy their music. Depending on which identities respondents felt motivated to align their responses with, some observed relationships may have appeared greater than they actually are.

Altogether, these limitations likely balance out for the cultural-preference networks and negatively bias the observed relationships between political issue concerns and partisan identity and ideology. Of course, these results should be replicated under other conditions, ideally using trace data or other records of actual behavior paired with surveys. This approach would resolve many of the concerns and limitations involved with relying on closed-ended survey responses.

Even so, this study presents essential first evidence contradicting the view of partisan cultural polarization. By using networks to map shared preferences and individual-level demographic features, we can see that Democrats and Republicans do not hold diverging cultural preferences across various domains, while such divisions do appear, as expected, in regards to political considerations. 


\section{References}

Bishop, B. (2009). The big sort: Why the clustering of like-minded america is tearing us apart. Houghton Mifflin Harcourt.

Bourdieu, P. (1984). Distinction: A social critique of taste (R. Nice, Trans.). Harvard UP.

Deichert, M. (2018). Partisan cultural stereotypes: The effect of everyday partisan associations on social life in the united states (Doctoral dissertation).

DellaPosta, D. (2020). Pluralistic collapse: The "oil spill" model of mass opinion polarization. American Sociological Review, 0003122420922989.

DellaPosta, D., Shi, Y., \& Macy, M. (2015). Why Do Liberals Drink Lattes? American Journal of Sociology, 120(5), 1473-1511. https: //doi.org/10.1086/681254

Hiaeshutter-Rice, D., Neuner, F. G., \& Soroka, S. (2019). Divided by culture: Partisan imagery and political evaluations.

Hoffman, M. A. (2019). The materiality of ideology: Cultural consumption and political thought after the american revolution. American Journal of Sociology, 125(1), 1-62.

Kapner, S., \& Chinni, D. (2019). Are your jeans red or blue? Shopping America's partisan divide. The Wall Street Journal.

Katz, J. (2016). 'Duck Dynasty'vs.'Modern Family': 50 Maps of the US Cultural Divide. The New York Times.

Levendusky, M. (2018). Americans, not partisans: Can priming american national identity reduce affective polarization? The Journal of Politics, 80(1), 59-70.

Margolis, M. F., \& Sances, M. W. (2017). Partisan differences in nonpartisan activity: The case of charitable giving. Political Behavior, 39(4), 839-864.

Martin, G. J., \& Webster, S. W. (2020). Does residential sorting explain geographic polarization? Political Science Research and Methods, $1-17$.

Mummolo, J., \& Nall, C. (2017). Why partisans do not sort: The constraints on political segregation. The Journal of Politics, 79(1), 45-59.

Mutz, D. C., \& Rao, J. S. (2018). The real reason liberals drink lattes. PS: Political Science \& Politics, 51(4), 762-767.

Why Obama-Trump swing voters like heavy metal. (2019). The Economist. https: / / www. economist. com / graphic- detail / 2019 / 11 / 16 / why-obama-trump-swing-voters-like-heavy-metal 


\section{Appendix A - Survey Questions}

1. What year were you born?

2. Which of the following best describes your gender?

- Man

- Woman

- Nonbinary

- Other (please specify)

3. Which of the following best describes your own background in racial and ethnic terms?

- African American

- White

- Hispanic or Latino

- Asian

- Native American or American Indian

- Middle Eastern

- Other (please specify)

4. Which of the following best describes the highest level of education you have completed?

- Some high school, but no degree

- High school degree

- Some college, but no degree

- Associate's or Bachelor's degree

- Some graduate school, but no degree

- Gradutae degree (e.g. MA, PhD, MD, or JD)

- Other (please specify)

5. Which of the following best describes your party affiliation?

- Strong Democrat

- Democrat

- Independent, but I lean Democrat

- Independent

- Independent, but I lean Republican

- Republican

- Strong Republican

6. Which of the following best describes your ideology?

- Very liberal

- Liberal

- Moderate, but I lean liberal

- Moderate

- Moderate, but I lean conservative

- Conservative

- Very conservative

7. Which are your three favorites among the following musicians?

- Childish Gambino

- Kacey Musgraves

- Dua Lipa 


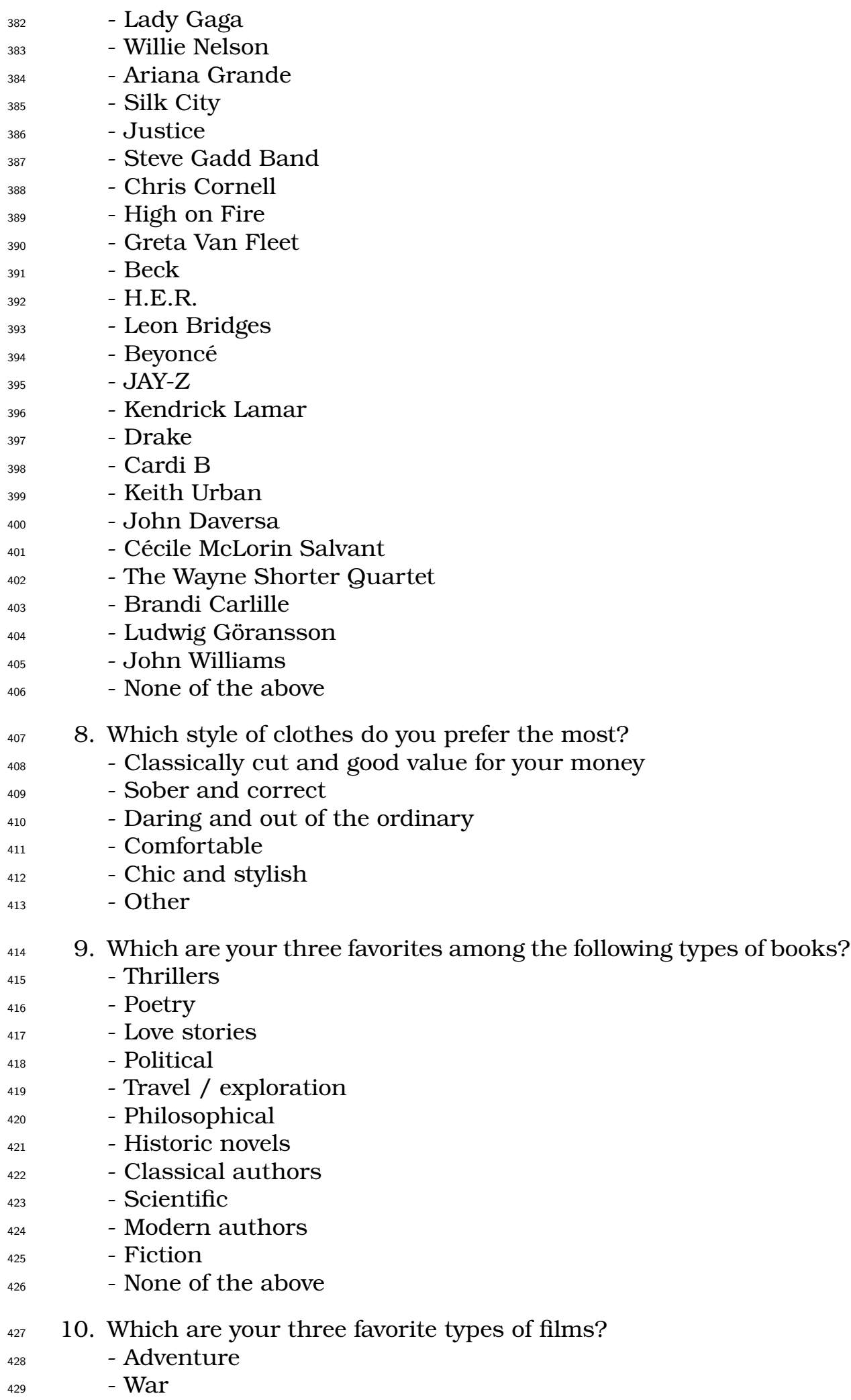




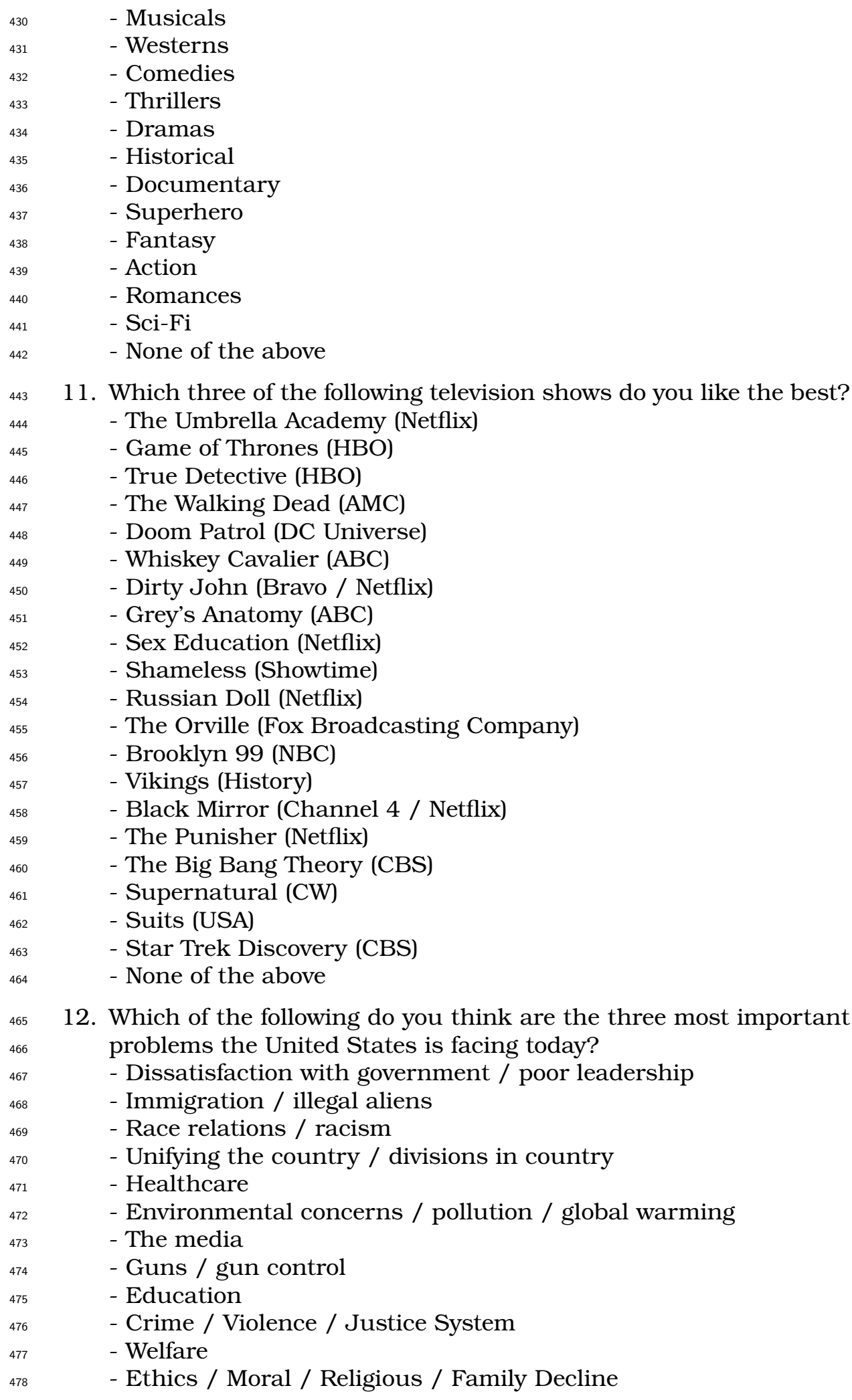




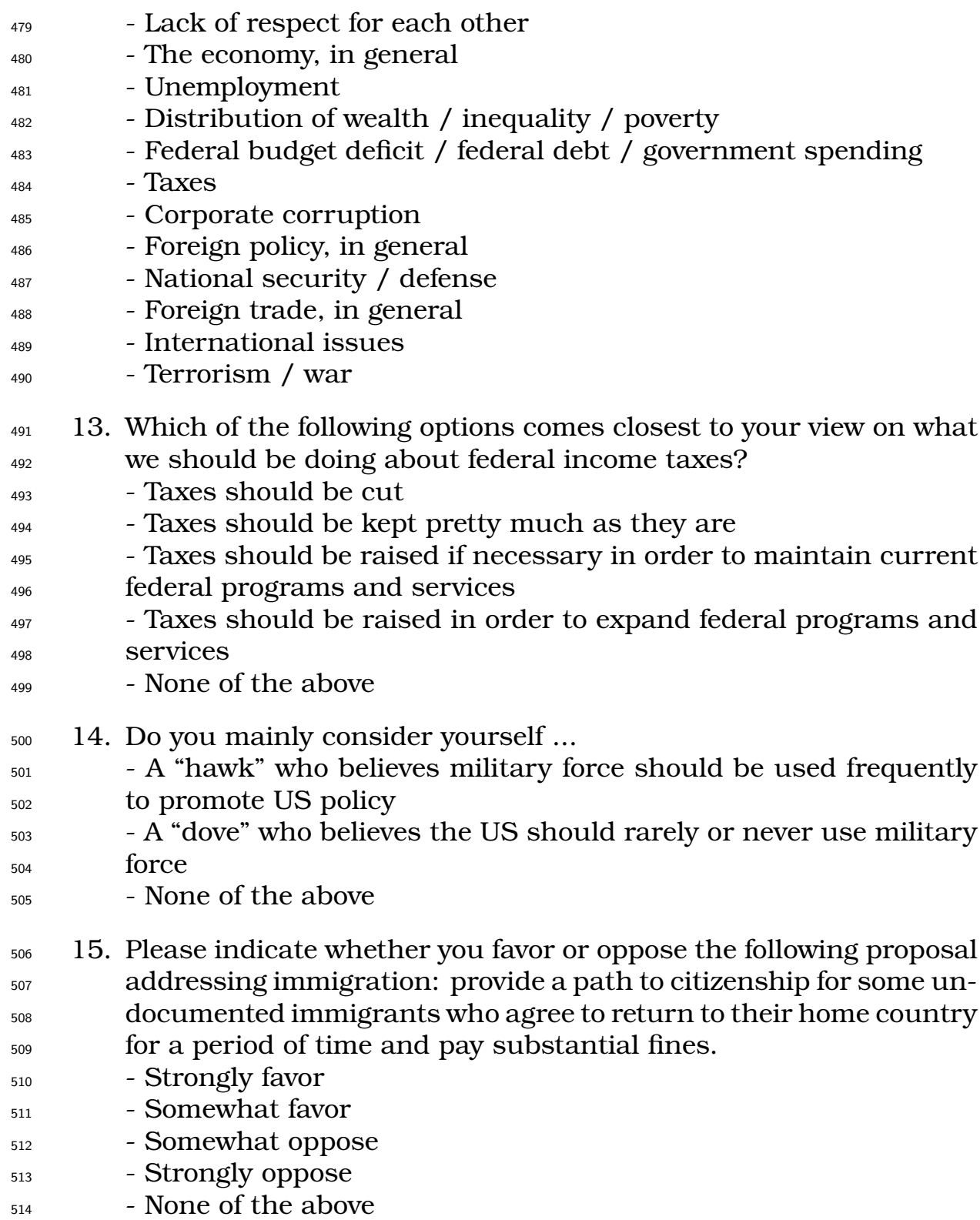




\section{Appendix B - Sample Details}

The survey sample studied here was collected in three waves of a larger study in Spring and Winter 2019 via Amazon Mechanical Turk.

The mean birth year among respondents was 1982, while the median was 1985. The interquartile range ran from 1976 to 1991.

The sample was relatively balanced in regards to gender. 681 respondents identified as men, 645 as women, 6 as nonbinary, and 1 as Other.

This relative balance was not repeated in regards to race. 939 of the respondents identified as white, 159 as African American, 100 as Asian, 96 as Latino, 28 as Other, 9 as Native American or American Indian, and 2 as Middle Eastern.

Similarly, educational attainment was not well balanced. 910 respondents reported achieving a college degree, while 423 did not.

Political identity was also slightly imbalanced. 158 respondents reported identifying as a Strong Democrat, 334 as Democrats, 167 as Independents that leaned Democrat, 235 as Independents, 126 as Independents that lean Republican, 231 as Republican, and 82 as Strong Republicans.

Ideological identity followed the same pattern. 180 reported being very liberal, 311 as liberal, as moderate, but leaning liberal, 254 as moderate, 131 as moderate leaning conservative, 201 as conservative, and 78 as very conservative. 